\title{
Appropriate Documentation, Billing and Coding in Interventional Pain Practice
}

\author{
Laxmaiah Manchikanti, MD
}

Appropriate documentation, billing and coding in interventional pain practice is a crucial issue with a wide arena of regulatory reforms. There have been reports of billions of dollars in losses in health care fraud. Office of Inspector General reports a massive war on health fraud. Substantial savings from prepayment audits for Part B in 1999, and continued criminal filings by the Department of Justice indicate persistence of Health Care Financing Administration to combat fraud. In addition President Clinton's initiatives to fight Medicare waste, fraud, and abuse have created increased fear of investigation or prosecution among physicians, leading to changes in their practice patterns.

Documentation of medical necessity with coding that correlates with multiple components of the patient's medical record, operative report, and billing statement is important.

Multiple issues of documentation, billing, and coding are facts of life for physicians practicing interventional pain management. Never in United States history has there been such an emphasis on the description and definition of what the physician does for and to the patient. Compliance with laws and regulations encompassing documentation, medical records, coding, billing, collections, contracts, auditing, and other areas are of crucial importance to today's interventional pain physician. The KennedyKassebaum Health Reform Bill of 1996 provided the Office of the Inspector General (OIG) and the Federal Bureau of Investigation with broad powers and directed them to identify and prosecute health care fraud and abuse. The Budget Reconciliation Act of 1997 empowered and directed the Heath Care Financing Administration (HCFA) to develop a payment system based upon physician work, and guideline documentation of that work. Thus, docu-

From Pain Management Center of Paducah, Paducah, KY. Dr. Manchikanti is medical director of Pain Management Center of Paducah. Address correspondence: Laxmaiah Manchikanti, MD, 2831 Lone Oak Road, Paducah, KY 42003
This review describes the regulatory issues, steps in documentation of medical necessity, appropriate billing and coding, and examples of codes describing CPT 1999 and 2000 for a multitude of procedures.

These illustrations and the information provide practical considerations for the use of interventional techniques in the management of chronic pain based on the current state of the art and science of interventional pain management, rules and regulations. However, this article and its descriptions do not constitute legal advice.

Keywords: Interventional pain management, medical necessity, billing, coding.

mentation, coding and billing are in the forefront of modern medicine. In addition to the Budget Reconciliation Act of 1997, and the Kennedy-Kassebaum Health Reform Bill of 1996, HCFA has also created the National Correct Coding Council (NCCC). It's purpose was to develop strategies for HCFA's Bureau of Program Operations to control improper coding that leads to inappropriate or increased payments in Part B claims. As a direct outgrowth of NCCC's work, HCFA established the National Correct Coding Policy in 1996 and eventually implemented the Medicare "correct coding" initiative to identify and isolate inappropriate coding, unbundling, and other irregularities in coding.

The HCFA reported overpayments of $\$ 12.6$ billion in 1998, and $\$ 13.5$ billion in 1999 in health fraud. Around the same time the OIG released a report that showed massive war on health fraud. As shown in Fig. 1, 22\% of total health care overpayments were attributed to physician practices in 1999. OIG also estimated more than half of rehab payments in 1997-1998 fiscal year were improper: either medically not necessary, poorly documented or not provided by licensed personnel. In addition, the HCFA has 
Fig. 1. Reported errors for Medicare program for 1999
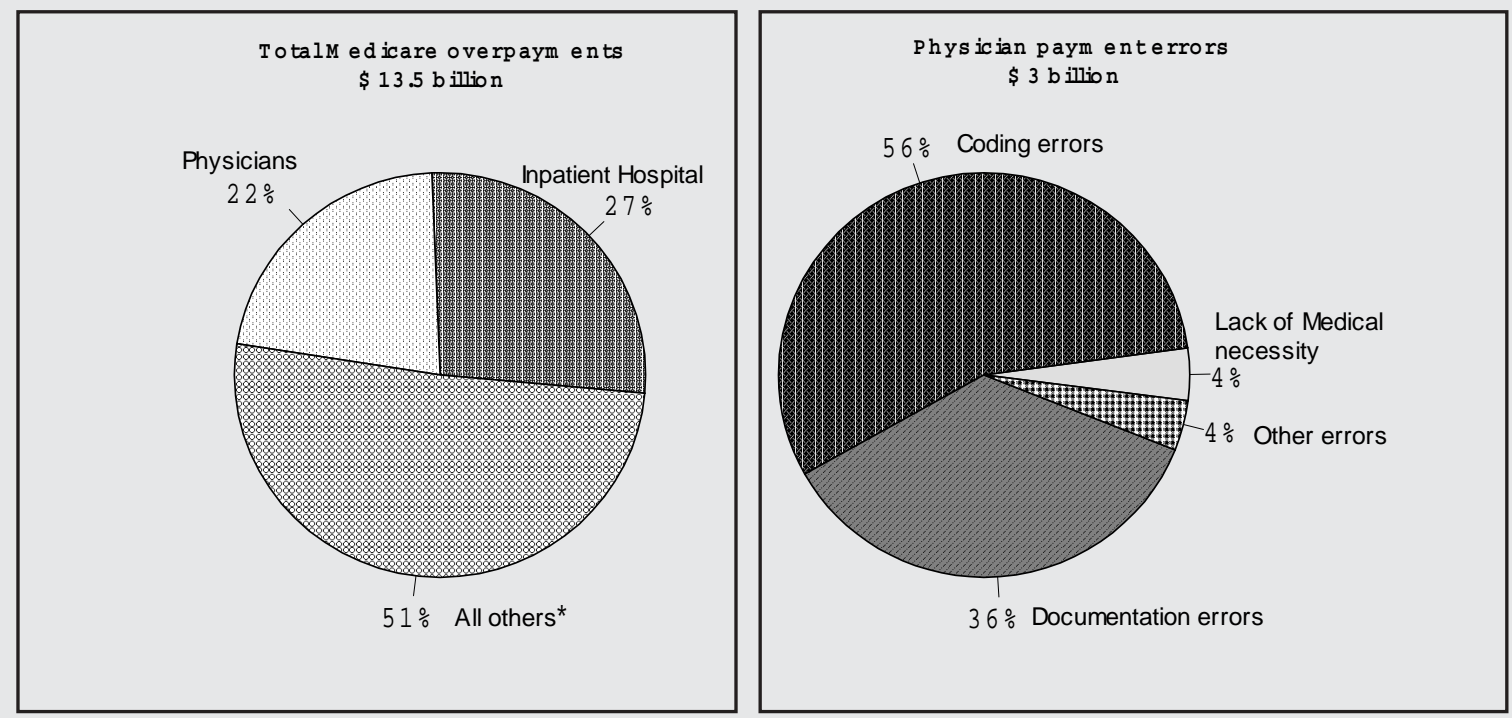

* All others include outpatient services, Home health agency, Durable Medical equipment Suppliers, etc.

Source: Office of Inspector General

recouped approximately $\$ 1.2$ billion in the first half of the fiscal year 1999 from Part B pre-payment audits alone from ambulatory surgery centers, hospital outpatient departments, physician claims, and durable medical equipment, among others. The government won $\$ 524,000,000$ last year from fines and settlements in health fraud cases, according the 1999 annual report on the Department of Justice - Health and Human Services (DOJ-HHS) Health Care Fraud and Abuse Control Program. Criminal filings by Department of Justice Prosecutors were up 16\% in 1999 from 363 in 1998 to 371 in 1999. By the end of 1999 US attorneys' offices had 2,278 civil health care fraud matters pending. In addition, the OIG also excluded 2,976 individuals and entities from federal health care programs in 1999. Tables 1 and 2 show Medicare exclusions and sanctions with sample monthly exclusion and sanction lists. President Clinton also proposed in the fiscal year 2001 budget many initiatives to fight Medicare waste, fraud, and abuse (1):

- Approximately $\$ 8$ billion in savings generated by trimming waste and Medicare payment policy

- Increased funds to government fraud programs and the Department of Health and Human Services - Office of Inspector General

- A new initiative to improve performance of Medicare contractors
- A fraud fighter in every Medicare contractor's office

- New technology to track false claims

The HCFA administrator Nancy-Ann Min DeParle states, it will:

- Strengthen contractor oversight

Table 1. Exclusions of medical providers

\begin{tabular}{lcc}
\hline Physician Specialty & $\begin{array}{c}\text { Sample of } \\
\text { Monthly } \\
\text { Exclusions }\end{array}$ & $\begin{array}{c}\text { Total } \\
\text { Exclusions }\end{array}$ \\
\hline Medical Practice & 35 & 2,883 \\
Chriopractic Practice & 21 & 1,290 \\
Psychology Practice & 8 & 322 \\
Podiatry Practice & 3 & 347 \\
Osteopathic Practice & 2 & 237 \\
Optometry Practice & 1 & 96 \\
\hline Total & $\mathbf{7 0}$ & $\mathbf{5 , 1 7 5}$ \\
\hline
\end{tabular}


Table 2. Sanctions of medical providers

\begin{tabular}{lcc}
\hline Sanction Type & $\begin{array}{c}\text { Sample of } \\
\text { Monthly } \\
\text { Exclusions }\end{array}$ & $\begin{array}{c}\text { Total } \\
\text { Exclusions }\end{array}$ \\
\hline $\begin{array}{l}\text { License revocation or } \\
\text { suspension } \\
\begin{array}{l}\text { Program-related } \\
\text { conviction }\end{array}\end{array}$ & 173 & 5,485 \\
$\begin{array}{l}\text { Patient abuse and } \\
\text { neglect }\end{array}$ & 65 & 5.403 \\
$\begin{array}{l}\text { Default on health } \\
\text { education loan } \\
\text { Sanctioned individual } \\
\text { owned entity }\end{array}$ & 23 & 2,258 \\
\hline Total & 6 & 620 \\
\hline
\end{tabular}

- Issue contractor report cards to compare performance standards

- Expand correct coding initiative

The HCFA says its goal is to reduce the improper payment rate to $5 \%$ or lower by fiscal year 2002, from $8 \%$ during fiscal year 1999 (fig. 2). It also intends to reduce fee-for-service (FFS) error rate to 6\% by October 2000 by:

- Updating software systems to keep pace with questionable billing practices

- Enhancing prepayment and post payment controls

- Pushing practices to maintain appropriate documentation and use proper coding

- Urging providers to maintain compliance plans

- Increasing number of carrier medical directors

- Implementing "program safeguards" for carriers that leave Medicare program

- Implementing carriers to recover improper payments discovered during audits

- Identifying high risk areas and reinstating surveillance initiators through peer review organizations

- Refining guidelines for proper coding and documentation

In a recent survey by the Association of American Physicians and Surgeons, physicians expressed their concerns

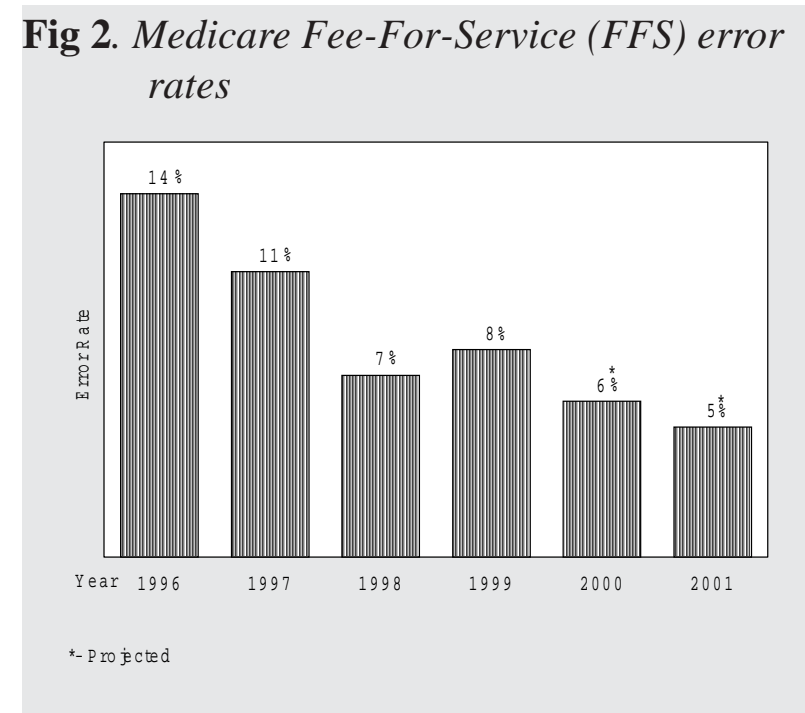

that the stepped-up threats of prosecution of doctors and government red tape may make it harder for Medicare beneficiaries to find doctors to treat them (1). According to this survey, $82 \%$ of physicians reported an increased fear of prosecution or investigation in the past 3 years, and $71 \%$ reported making changes in their practice to avoid the threat of prosecution, including restricting services. Sixty six percent plan to retire from patient care at an earlier age than they would have considered 5 years ago, $23 \%$ of the respondents no longer accept new Medicare patients, and 34\% reported having difficulty finding referrals for their Medicare patients. The HCFA also increased attention focused on getting beneficiaries and the public to report Medicare fraud has resulted in increased cost of OIG's fraud hotline (Fig. 3).

In a recent case of charges of up-coding the federal government is now seeking as much as $\$ 37$ million from a physician for allegedly over-billing the civilian health and

Fig 3. Medicare fraud hot line hits

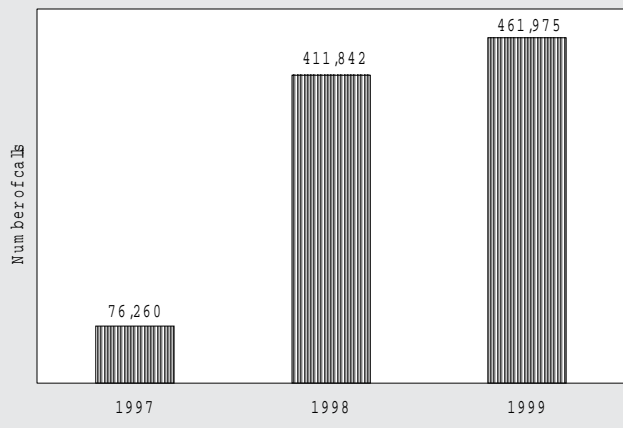


medical program of the uniformed services for treatment provided to patients in general practice (2). In some recent high-profile cases, Fresenius Medical Care, Lexington, Massachusetts, agreed to pay the government \$486 million to settle fraudulent billing and kickback charges against a company acquired in 1996. Similarly, Beverly Enterprises, Inc., of Fort Smith, Arkansas, also agreed to pay the US Government $\$ 170$ million in restitution plus a $\$ 5$ million fine to settle civilian criminal allegations of a nationwide scheme to defraud Medicare from 1992 to 1998. Consequently, whistle blowers in Fresenius settlements will take home a staggering $\$ 65.8$ million from the civil recovery settlement. An anesthesia group settled for $\$ 3.2$ million for insufficient supervision, and an emergency physician group settled for $\$ 15$ million for upcoding. This is not surprising considering that Qui Tam lawsuits increased 74\% in 1997. Table 3 shows Qui Tam cases and recoveries. In 1999 work plan, the OIG is targeting $600,000+$ physicians in practice.

\section{DOCUMENTATION OF MEDICAL NECESSITY}

Medical necessity requires appropriate ICD-9-CM diagnosis codes with their distinct rules for Volumes I and II to justify services rendered and indicate the severity of the patient's condition (3). The Balanced Budget Act (HR 2015, Section 4317) requires all physicians to provide the diagnostic information for all Medicare/Medicaid patients starting from January 1, 1998, failure of which can result in prosecution (4). The International Classification of Diseases, also known as ICD-9-CM, is a system used to document complaints, symptoms, conditions, problems, and diagnosis for an encounter or procedure. Physicians should code by listing the ICD-9-CM diagnostic codes shown in the medical record to be chiefly responsible for the services provided. Coding should be to the highest degree of certainty for each encounter and each interventional pain procedure. Coding also should correlate with multiple components the of patient's medical record including initial evaluation or history and physical, operative report or procedure note, and also the billing statement. However, chronic conditions treated on an ongoing basis, such as in an interventional pain management setting, may be reported as many times as the patient receives treatment and care for the condition. If proper diagnosis is not established, codes that describe symptoms and signs, as opposed to the diagnosis, are acceptable for reporting purposes until the diagnosis is confirmed.

There is significant confusion with regards to what constitutes appropriate documentation and provides medical necessity in interventional pain management. The arena of medical record documentation is similar to evaluation and management services though there are some differences. History and physical during the first visit is the same as described for evaluation and management services in pain management (4). Once the initial evaluation is performed, a history and physical may be developed in a simpler format showing the pertinent facts and important issues as required by law, as well as either policies of practice or institutional policies such as surgery centers and hospitals. History and physical has to be updated each 30 days for repeat visits and repeat interventional pain pro-

Table 3. Qui Tam Cases and Recoveries

\begin{tabular}{lccc}
\hline & FY & FY & FY \\
& 1996 & 1997 & 1998 \\
\cline { 2 - 3 } & 346 & 546 & 469 \\
Qui Tam cases filed & 176 & 306 & $\$ 261$ \\
Qui Tam cased filed alleging health care fraud & $\$ 66$ & & $\$ 608$ \\
$\begin{array}{l}\text { Health-care fraud judgments/settlements in matters with Qui Tam } \\
\text { claims (millions) }\end{array}$ & & $\$ 961$ & $\$ 300$ \\
Total health-care fraud judgments/settlements (millions) & $\$ 136$ & & \\
\hline
\end{tabular}

Source: Department of Justice 
cedures. If the first encounter for an interventional pain procedure is within 30 days of initial evaluation, the initial evaluation can be used and will meet all the criteria for history and physical. However, subsequently history and physical should be updated every 30 days if the procedures are repeated. The history and physical should include the following information.

\section{History}

Chief Complaint: Purpose of patient visit.

History of Present Illness: History of present illness may list the symptomatology for follow-up visits, describing as many elements as possible, including location, quality, severity, duration, timing, context, modifying factors and associated signs and symptoms.

Past, Family, and Social History: The past, family, and social history criteria must be documented. Follow-up criteria may be met by describing any new information or noting there has been no change in the information, and noting that the date and location of earlier review of past, family, and social history.

Review of Systems: Review of systems should include constitutional, cardiovascular, respiratory, gastrointestinal, musculoskeletal, neurological, and allergies. However, a statement should be made regarding the other systems if they are normal; if they are not, abnormalities should be described. All other systems may be documented by stating that they were normal; however, for follow-ups one can also choose to meet the criteria and document by describing any new information or noting there has been no change in the information; and noting that the date and location of earlier review of systems.

\section{Physical Examination}

The physical examination should include constitutional evaluation with height and weight and general appearance, musculoskeletal and neurological examination, and examination of heart and lungs.

\section{Medical Decision Making}

Review of Investigations: This may consist of review of the investigations, which may be documented similar to review of systems and past family and social history or these can be documented on the history and physical.
Impression: Impression with all diagnoses should be listed.

Procedure(s): Procedures to be performed should be listed.

After completing the procedure, further documentation, is required generally on a separate note, which should include the following:

- Chief complaint or reason for the visit;

- The quality and duration of pain relief if this was a repeat procedure;

- Any side effects associated with the previous pro cedure, if this is a repeat procedure;

- Discussion and plan of the above procedure;

- Discussion of the complications associated with the procedure;

- Discussion of the other aspects of the manage ment; and

- Procedure note describing the technique, etc. in brief.

\section{Discharge/Disposition}

Discharge/disposition should indicate the condition of the patient at the time of the discharge and return appointment, as well as discharge instructions.

\section{Operative Note}

An operative note is required by law if the procedure is performed in the operating room, either in a surgical center or hospital. This note should be more detailed than the follow-up note described earlier. The documentation should include:

1. Identifying information of the patient;

2. Names of nurse(s), surgical technician(s), radiological technician(s), etc;

3. Diagnoses;

4. Procedure or procedures performed;

5. Indications/medical necessity;

6. Preoperative preparation;

7. Intraoperative information;

8. Postoperative recover; and

9. Discharge/disposition.

\section{Indications/Medical necessity}

It is best if indications and medical necessity are described separately for Medicare and Medicaid, even though most 
records do document these things in various parts of the medical record. Documentation at this level is much more advantageous, for the physician, billing personnel, and investigators. This should include:

- Symptoms of the patient with diagnosis itemized into:

Each physical complaint with
diagnosis;
Medical problems with diagnosis;
Psychological problems with diagnosis;
The effect of pain on physical,
functional, and psychological status,
and activities of daily living, with its
effect on quality of life;
Effect of previous treatment(s), includ-
ing all conservative modalities of
treatments with drug therapy, physical
therapy, chiropractic management,
massage therapy, etc all interventional
procedures, and surgical procedures;
A statement to the effect that there is no
significant evidence of intraspinal
obstruction for any procedure on the
spine;
For repeat procedures, description of the
quality and duration of relief with its
resultant effect on functional status, as
well as activities of daily living;
Reasons for repeating the injection
therapy; generally, the return of pain
with some deterioration, with expecta-
tion of further deterioration in physical
or functional status.

Manchikanti and colleagues (8) defined indications and medical necessity for interventional pain procedures.

\section{Preoperative}

This should describe informed consent and preparation of the patient prior to the procedure including intravenous access, and sedation, etc.

\section{Intraoperative}

Documentation consists of:

- Sedation and monitoring,

- Appropriate positioning,
- Sterile field preparation, and

- Description of the procedure with needle placement, contrast injection findings, and injection of other drugs.

\section{Post-operative}

Documentation consists of:

- Monitoring,

- Complications,

- Further treatment carried out in the postoperative phase.

\section{Discharge/Disposition}

Documentation consists of:

- Status at discharge, and

- Discharge instructions.

\section{Billing and Coding}

Billing and coding not only requires ICD-9-CM but also CPT-4 ${ }^{\mathrm{TM}}$, either 1999 or 2000 (4-8).

Physicians' current procedural terminology (CPT) is an up-to-date, comprehensive classification and nomenclature system for accurately identifying procedures and services by physicians and other health care professionals. At this point, interventional pain management is complicated by various issues as described in interventional pain management coding in the new millennium (7). The CPT 2000 comprises numerous new codes of interest to interventional pain specialists, along with revisions of some old codes with result and deletion of multiple old codes (5-8). Thus, coding and billing described herewith will include both 1999 codes, as well as 2000 codes. Two documents entitled, "Interventional Techniques in the Management of Chronic Pain:" Part 1.0 by Manchikanti et al (8) and "2000 Common Coding Scenarios for Spine Procedures and Injection Techniques" by the North American Spine Society (9) describe diagnostic and procedure coding in interventional pain management. This article manuscript will describe only commonly performed injection procedures and coding guidelines for the same.

\section{CORRECT CODING}

For purposes of correct coding, interventional pain physicians and their offices should be familiar with the medical/surgical practice and packages, modifiers, separate 
procedures, comprehensive and component codes, incorrect coding and/or unbundling $(10,11)$.

\section{Medical Surgical Practice and Packages}

As a general principle, all services integral to accomplishing a procedure are considered to be included in a procedure and, therefore, are a component part of comprehensive code. These include intravenous access, cardiopulmonary monitoring, and management of complications.

\section{Modifiers}

An essential part of the CPT coding system is the utilization of modifiers that expand the information provided by the CPT codes. Use of modifiers indicates that the services were performed under circumstances which did not involve "unbundling."

\section{Separate Procedures}

The classification of a procedure as a "separate procedure" indicates that the procedure, while it may be performed separately, is generally included in a more comprehensive procedure and that the service may not be reported when a related, more comprehensive service is performed. Hence, when a related procedure is performed, a code with a designation of "separate procedure" may not be reported with the primary procedure. The "separate procedure" designation frequently is met with controversy in interventional pain management procedures, including radiological procedures.

The most common interventional pain management procedures with a designation of "separate procedure" in 1999 included:

1. 62289 - lumbar or caudal epidural steroids,

2. 62278 - lumbar or caudal epidural with local anesthetic,

3. 62288 - subarachnoid injection,

4. 62298 - cervical or thoracic epidural steroids, and

5. 62275 - cervical or thoracic epidural steroids with local anesthetic

However, fortunately, the new epidural codes from CPT 2000: 62310, 62311, which replace the 1999 codes, are not classified as separate procedures. However, one should exercise caution in taking a liberal position, as CPT 2000 codes have much better definitions and descriptions, thus avoiding any opportunities for unbundling.

\section{Comprehensive and Component Codes}

Comprehensive codes include certain defined services that are separately identifiable by other codes known as component codes. Because component codes are captured by comprehensive codes, they may not be listed separately when the complete procedure is done. Relevant comprehensive codes and component codes of interventional pain management have been compiled (10). Although no such codes have been developed for the year 2000, these are expected to be released in April of the year 2000, which probably will include many interventional pain procedures.

Additionally, black box edits are secret code pairs, used by carriers to deny claims without explanation (12). These are similar to comprehensive/component or mutually exclusive codes, but providers are not told why the claims are denied, and denials can be used for audit. The HCFA plans to implement 305 new black box code pairs by April 1, 2000, leading to total number of these edits to 1,288. However, Medicare Payment Advisory Commission (Med Pac) recommended that "black box" edits be made public.

\section{Incorrect Coding and/or Unbundling}

Unbundling is the billing of multiple procedure codes for a group of procedures that are covered by a single comprehensive code. The unbundling may be intentional or unintentional. Unbundling has been described as follows:

- Fragmenting service into component parts and billing each component part as if it were a separate service.

- Reporting separate codes for related services when one comprehensive code includes all related services.

- Reporting two codes for bilateral procedures when one code is appropriate.

- Down coding a service in order to use an additional code when one higher level, more comprehensive code is appropriate.

\section{Cautionary Note on CPT 2000}

Some of the new codes in interventional pain management include (5):

1. 76005 - fluoroscopy guidance and localization,

2. 72275 - epidurography, and

3. 73542 - sacroiliac joint arthrography. 
The CPT 76005 is described as follows: fluoroscopy guidance and localization of needle or catheter tip for spine or paraspinous diagnostic or therapeutic injection procedures (epidural, transforaminal epidural, subarachnoid, paravertebral facet joint, paravertebral facet joint nerve or sacroiliac joint), including neurolytic agent destruction. Thus, if fluoroscopy is used this code may be used either with epidural or subarachnoid injection procedures with CPT codes 27096,62270 to 62273,62280 to 62282,62310 to 62319,64470 to 64476,64479 to 64484,64600 to 64620 , and 64622 to 64627 . Hence, this code may not be used with percutaneous lysis of epidural adhesions (CPT 62263), which includes radiologic localization and contrast administration; with cervical/thoracic discography (CPT 62291) or lumbar discography (CPT 62290). This code may not be used with 64630, 64640, 64680, epidurography code 72275 , or 73542 - sacroiliac joint arthrography.

The CPT 72275 - epidurography differs from fluoroscopy guidance and localization of needle or catheter tip CPT 76005. Similarly, CPT 73542 - sacroiliac joint arthrography also differs from 76005, as it represents a formal contrast study that includes fluoroscopy as with epidurography. Thus, 76005 - fluoroscopy guidance, 72275 epidurography, 73542 - sacroiliac joint arthrography, 72285 - discography, cervical or thoracic, and 72295 discography, lumbar, are not stand-alone codes and should always be used with appropriate procedural codes. In addition, either in case multiple injections or multiple attempts are made, fluoroscopy guidance code 76005 can be used only once.

\section{Frequency and Number of Injections or Interventions}

- In the diagnostic and stabilization phase, a patient may receive injections at intervals of no sooner than 1 week and preferably 2 weeks for most types of blocks except for blockade in cancer pain or when a continuous administration of sympathetic blocks is employed (8).

- In the treatment phase (after the stabilization is completed), the frequency of interventional techniques should be 2 months or longer between each injection provided that at least $>50 \%$ relief is obtained for 6 weeks (6). However, if the neural blockade is applied for different $\mathrm{r}$ e $\mathrm{g}$ i o $\mathrm{n} \mathrm{s}$, they can be performed at intervals of no sooner than 1 week and preferably 2 weeks for most type of blocks. The therapeutic frequency must re- main 2 months for each region. It is further suggested to treat all regions at the same time provided all procedures are performed safely (8).

- In the stabilization phase, the number of injections should be limited to no more than four times per year in cases of all the blocks except sympathetic blocks, in which case six times should be reasonable.

- In the maintenance phase, the interventional procedures should be repeated only as necessary judging by the medical necessity criteria and these should be limited to a maximum of six times for local anesthetic and steroid blocks and four times for interventions such as radiofrequency thermoneurolysis, and cryoneurolysis for a period of one year.

- For percutaneous non-endoscopic adhesiolysis with a 3-day protocol, 2-3 interventions per year are recommended; with a 1-day protocol, a maximum of 6 times per year is recommended.

- For endoscopic adhesiolysis, it is recommended that there be no more than 2-3 inter ventional procedures per year.

- Under unusual circumstances with a recurrent injury, carcinoma, cervicogenic headache, or regional sympathetic dystrophy, blocks may be repeated at intervals of 6 weeks after stabilization in the treatment phase.

\section{Combination of Blocks/Interventions}

It may be essential to combine, in certain circumstances, more than one block (8). This may include an epidural for the cervical region and facet-joint blocks for the lumbar region; epidural and facet-joint blocks for the same region in case of identification of pain generators from both sources; a sympathetic block and facet-joint block if there are two different sources of pain or if two different regions are affected in combination with trigger-point injections (8). Consequently, blocks also may be combined with other interventional techniques.

\section{Number Per Setting}

It is recommended that a physician should consider a patient in totality and treat multiple regions of the patient in the same setting, as long as it is safe and feasible (8). Attempts to treat one particular organ at a different time are not an absolute necessity (8). 
However, no more than five procedures (different procedures and/or multiples of one procedure - or total line items or procedures) must be billed in one setting for any of the following: the procedures are performed in different regions or a combination of procedures in one or multiple regions. For treatment of a single region (eg, only lumbosacral spine or cervical spine) a maximum of four procedures (different procedures and/or multiples of one procedure - or total line items or procedures) should be billed.

\section{FACET JOINT BLOCKS AND NEUROLYSIS}

\section{Procedure (CPT) Codes}

\section{Codes (6)}

- 64442 - Injection, anesthetic agent; paravertebral facet-joint nerve, lumbar, single level

- 64443 - Injection, anesthetic agent; paravertebral facet joint nerve, lumbar, each additional level

64622 - Destruction by neurolytic agent, paravertebral facet joint nerve, lumbar, single level

64623 - Destruction by neurolytic agent, paravertebral facet joint nerve, lumbar, each additional level

2000 Codes (5)

- 64470 - Injection, anesthetic agent and/or steroid, paravertebral facet joint or facet joint nerve, cervical or thoracic $(\mathrm{C} / \mathrm{T})$, single level

- 64472 - Injection, anesthetic agent and/or steroid, paravertebral facet joint or facet joint nerve, cervical or thoracic $(\mathrm{C} / \mathrm{T})$, each additional level

- 64475 - Injection, anesthetic agent and/or steroid, paravertebral facet joint or facet joint nerve, lumbar or sacral (L/S), single level

- 64476 - Injection, anesthetic agent and/or steroid, paravertebral facet joint or facet joint nerve, lumbar or sacral (L/S), each additional level

-64626-Destruction by neurolytic agent, paravertebral facet joint nerve; cervical or thoracic $(\mathrm{C} /$ $\mathrm{T})$, single level

- 64627 - Destruction by neurolytic agent, paravertebral facet joint nerve; cervical or thoracic $(\mathrm{C} /$ $\mathrm{T})$, each additional level

64622 - Destruction by neurolytic agent, paravertebral facet joint nerve; lumbar or sacral (L/S), single level
64623 - Destruction by neurolytic agent, paravertebral facet joint nerve; lumbar or sacral $(\mathrm{L} / \mathrm{S})$, each additional level

- 27096 - Injection procedure for sacroiliac joint, arthrography and/or anesthetic/steroid

- 73542 - Radiological examination, sacroiliacjoint arthrography, radiological supervision and interpretation

76005 - Fluoroscopic guidance and localization or needle or catheter tip for spine or paraspinous diagnostic or therapeutic procedures

\section{Examples}

i. Cervical facet joint injection, single level (C5/6 joint)

\section{Codes:}

$64470-\mathrm{C} / \mathrm{T}$ facet joint injection, single level

76005 - Fluoroscopic guidance

1999 Codes:

No specific code available (lumbar code is used)

64442 - Facet joint nerve injection, single level

ii. Multiple cervical facet joint injections (C4/5 through $\mathrm{C} 6 / 7$ )

2000 Codes:

$64470-\mathrm{C} / \mathrm{T}$ facet joint injection, single level

64472-51 (two units) - C/T facet joint injection, subsequent levels

76005 - Fluoroscopic guidance 1999 Codes:

No specific codes are available (lumbar codes are used)

64442 - Facet joint nerve block, single level

64443-51 (two units) - Facet joint nerve blocks, subsequent levels

iii. Bilateral cervical facet joint injections $(\mathrm{C} 4 / 5$ through C6/7)

2000 Codes:

64470-50 - C/T facet joint injection, single level ( $\mathrm{C} 4 / 5$ bilateral)

64472-50 - C/T facet joint injection, each additional level (C5/6 bilateral) 


\section{Codes:}

76005 - Fluoroscopic guidance

No specific codes are available (lumbar codes are used bilateral billing not available with 1999 CPT codes)

$64442-$ Facet joint nerve block,
single level
64443-51 (two units) - Facet joint
nerve blocks, subsequent levels

iv. Cervical facet joint nerve blocks, single level (C5/6 joint - C5 and C6 medial branch nerves) 2000 Codes:
$\checkmark \quad 64470-\mathrm{C} / \mathrm{T}$ facet joint nerve block, single level (C5/6 joint)
76005 - Fluoroscopic guidance

1999 Codes:

No specific codes are available (lumbar codes are used)
- 64442 - Facet joint nerve block, single level

v. Cervical facet joint nerve blocks, multiple levels (C3/4 and $\mathrm{C} 4 / 5$ joints $-\mathrm{C} 3, \mathrm{C} 4$, and C5 medial branch nerves)

\section{Codes:}

- $64470-\mathrm{C} / \mathrm{T}$ facet joint nerve block, single level (C3/4 joint)

- 64472-51 - C/T Facet joint nerve blocks, each additional level (C4/5 joint)

\section{$\checkmark 76005$ - Fluoroscopic guidance}

\section{Codes:}

No specific codes are available (lumbar codes are used)

$64442-$ Facet joint nerve block,
single level
64443-51 (two units) - Facet joint
nerve blocks, subsequent levels

vi. Cervical facet joint nerve blocks, multiple levels (C3/4 through C6/7 joints - C3 through C7 me dial branches)

\section{Codes:}

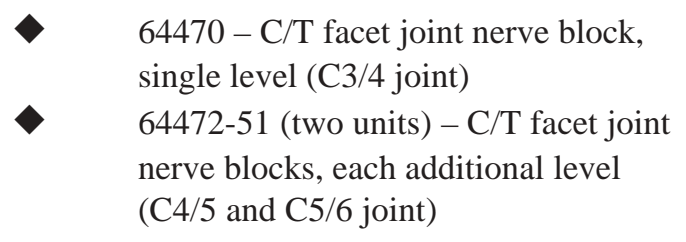

\section{$\checkmark \quad 76005$ - Fluoroscopic guidance 1999 Codes:}

No specific codes are available (lumbar codes are used)

$64442-$ Facet joint nerve block,
single level
64443-51 (two units) - Facet joint
nerve blocks, subsequent levels

vii. Bilateral cervical facet joint nerve blocks (C5/6 and $\mathrm{C} 6 / 7$ joints - $\mathrm{C} 5$ through $\mathrm{C} 7$ facet joint nerves or medial branch nerves)

\section{Codes:}

- 64470-50-C/T facet joint nerve blocks, single level (C5/6 joint bilateral)

- 64472-50 - C/T facet joint nerve blocks, each additional level (C6/7 joint bilateral)

\section{Codes:}

76005 - Fluoroscopic guidance

No specific codes are available (lumbar codes are used bilateral billing not available with 1999 CPT codes)

$$
\begin{aligned}
& 64442-\text { Facet joint nerve block, } \\
& \text { single level } \\
& \text { 64443-51 (two units) - Facet joint } \\
& \text { nerve blocks, subsequent levels }
\end{aligned}
$$

viii. Thoracic facet joint injection, single level (T5/6 joint)

\section{Codes:}
$\checkmark \quad 64470-\mathrm{C} / \mathrm{T}$ facet joint injection, single level (T5/6)
$\checkmark 76005$ - Fluoroscopic guidance

\section{Codes:}

No specific codes are available (lumbar codes are used)

$64442-$ Facet joint nerve block,
single level

ix. Multiple thoracic facet joint injections (T4/5 through T6/7)

2000 Codes:

$$
\begin{aligned}
& 64470-\mathrm{C} / \mathrm{T} \text { facet joint injection, } \\
& \text { single level (T4/5) } \\
& \text { 64472-51 (two units) - C/T facet joint } \\
& \text { injections, each additional level (T5/6 } \\
& \text { and T6/7) }
\end{aligned}
$$




\section{Codes:}

76005 - Fluoroscopic guidance

No specific codes are available (lumbar codes are used)

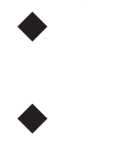

64442 - Facet joint nerve block, single level

64443-51 (two units) - Facet joint

nerve blocks, subsequent levels

x. Bilateral thoracic facet joint injections (T4/5 through T6/7)

\section{Codes}

\section{- 64470-50 - C/T facet joint injection, single level (T4/5 bilateral) \\ $\checkmark \quad 64472-50-\mathrm{C} / \mathrm{T}$ facet joint injection, each additional level (T5/6 bilateral) \\ 1999 Codes: \\ 76005 - Fluoroscopic guidance}

No specific codes are available (lumbar codes are used, however, bilateral billing is not available with 1999 CPT codes)

- 64442 - Facet joint nerve block, single level

64443-51 (two units) - Facet joint nerve blocks, subsequent levels

xi. Thoracic facet joint nerve blocks, single level (T5/6 joint - T5 and T6 medial branch nerves) 2000 Codes:

$$
\begin{aligned}
& 64470-\mathrm{C} / \mathrm{T} \text { facet joint nerve block, } \\
& \text { single level (T5/6 joint) } \\
& 76005-\text { Fluoroscopic guidance }
\end{aligned}
$$$$
1999 \text { Codes: }
$$

No specific codes are available (lumbar codes are used)

$$
\begin{aligned}
& 64442-\text { Facet joint nerve block, } \\
& \text { single level }
\end{aligned}
$$

xii. Thoracic facet joint nerve blocks, multiple lev els (T3/4 and T4/5 joints - T3, T4, and T5 me dial branch nerves)

\section{Codes:}

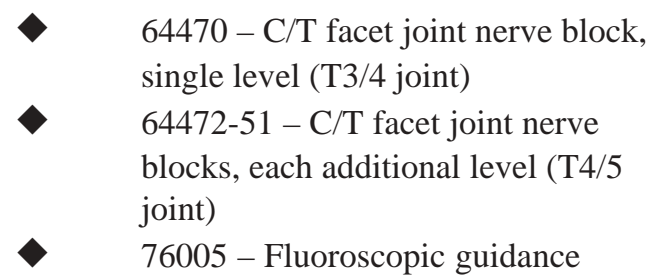

1999 Codes:

No specific codes are available (lumbar codes are used)

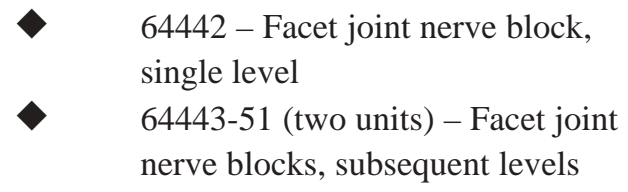

xiii. Thoracic facet joint nerve blocks, multiple lev els (T3/4 through T6/7 joints - T3 through T7 medial branches)

\section{Codes:}

$$
\begin{aligned}
& 64470-\mathrm{C} / \mathrm{T} \text { facet joint nerve block, } \\
& \text { single level (T3/4 joint) } \\
& 64472-51 \text { (two units) - C/T facet joint } \\
& \text { nerve blocks, each additional level } \\
& \text { (T4/5 and T5/6 joint) } \\
& 76005-\text { Fluoroscopic guidance }
\end{aligned}
$$

\section{Codes:}

No specific codes are available (lumbar codes are used)

$$
\begin{aligned}
& 64442-\text { Facet joint nerve block, } \\
& \text { single level } \\
& \text { 64443-51 (two units) - Facet joint } \\
& \text { nerve blocks, subsequent levels }
\end{aligned}
$$

xiv. Bilateral thoracic facet joint nerve blocks (T5/6 and $\mathrm{T} 6 / 7$ joints - $\mathrm{T} 5$ through $\mathrm{T} 7$ facet joint nerves or medial branch nerves)

\section{Codes:}

- 64470-50-C/T facet joint nerve block, single level (T5/6 joint bilat eral)

$\checkmark \quad 64472-50-\mathrm{C} / \mathrm{T}$ facet joint nerve blocks, each additional level (T6/7 joint bilateral)

\section{Codes:}

76005 - Fluoroscopic guidance

No specific codes are available (lumbar codes are used, however, bilateral billing is not available with 1999 CPT codes)

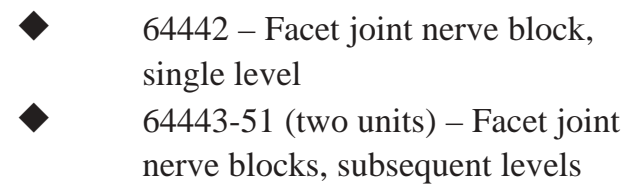

xv. Lumbosacral facet joint injection, single level (L3/4 joint) 


\section{Codes:}

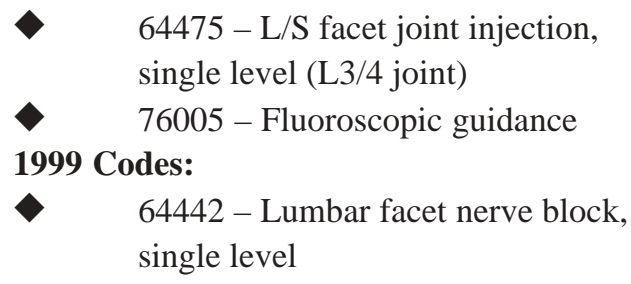

xvi. Lumbosacral facet joint injection, multiple lev els (L2/3 through L5/S1)

\section{Codes:}

64475 - L/S facet joint injection,
single level (L2/3 joint)
64476-51 (two units) - L/S facet joint
injection, each additional level (L3/4,
L4/5, and L5/S1 joints)
76005 - Fluoroscopic guidance
1999 Codes:
64442 - Lumbar facet joint nerve
block, single level
66443-51 (two units) - Lumbar facet
joint nerve blocks, subsequent levels

xvii. Bilateral lumbosacral facet joint injection (L3/4 and $L 4 / 5$ joints)

\section{Codes:}

$$
\begin{aligned}
& 64475-50-\text { L/S facet joint injection, } \\
& \text { single level (L3/4 joint bilateral) } \\
& 64476-50-\text { L/S facet joint injection, } \\
& \text { each additional level (L4/5 joint } \\
& \text { bilateral) } \\
& 76005-\text { Fluoroscopic guidance }
\end{aligned}
$$

1999 Codes:

Bilateral billing not allowed with 1999 CPT codes

$\checkmark \quad 64442$ - Lumbar facet joint nerve block, single level

64443-51 (two units) - Lumbar facet joint nerve blocks, subsequent levels

xviii. Lumbar facet joint nerve blocks, single level (L3/4 joint - L2 and L3 medial branch nerves or facet joint nerves)

\section{Codes:}

- $64475-\mathrm{L} / \mathrm{S}$ facet joint nerve block, single level (L3/4 joint)

$\checkmark \quad 76005$ - Fluoroscopic guidance 1999 Codes:

64442 - Lumbar facet nerve block, single level

xix. Multiple lumbar facet joint nerve blocks (L3/4 through L5/S1 joints - L2 through L4 medial branch nerves and L5 dorsal ramus)

2000 Codes:

- $64475-\mathrm{L} / \mathrm{S}$ facet joint nerve block, single level (L3/4 joint)

64476-51 (two units) - L/S facet joint nerve blocks, each additional level (L4/5 and L5/S1 joints)

$\checkmark \quad 76005$ - Fluoroscopic guidance 1999 Codes:

- 64442 - Lumbar facet joint nerve block, single level

- 64443-51 (two units) - Lumbar facet joint nerve blocks, subsequent levels

xx. Bilateral lumbar facet joint nerve blocks (L4/5 and L5/S1 joints - L3 and L4 medial branch nerves and L5 dorsal ramus)

2000 Codes:

- 64475-50 - L/S facet joint nerve block, single level (L4/5 joint bilateral)

- 64476-50 - L/S facet joint nerve blocks, each additional level (L5/S1 joint bilateral)

$\checkmark 76005$ - Fluoroscopic guidance

1999 Codes:

- $\quad 64442$ - Lumbar facet joint nerve block, single level

- 64443-51 (two units) - Lumbar facet joint nerve blocks, subsequent levels

xxi. Cervical paravertebral facet joint nerve neuroly sis (C5/6 joint - C5 and C6 medial branch nerves) 2000 Codes:

$\checkmark \quad 64626-\mathrm{C} / \mathrm{T}$ facet neurolysis, single level (C5/6 joint)

$\checkmark \quad 76005$ - Fluoroscopic guidance

\section{Codes:}

No specific codes are available (lumbar neurolysis codes are used)

64622 - Facet joint neurolysis, single level

xxii. Multiple cervical paravertebral facet joint neu rolysis (C4/5 through $\mathrm{C} 6 / 7$ joints - $\mathrm{C} 4$ through C7 medial branches) 


\section{Codes:}

64626 - C/T facet joint neurolysis,
single level (C4/5 joint)
64627-51 (two units) - C/T facet joint
neurolysis, each additional level (C5/6
and C6/7 joints)
76005 - Fluoroscopic guidance
1999 Codes:

No specific codes are available (lumbar neurolysis codes are used)

$64622-$ Facet joint neurolysis, single
level
$64623-51$ (two units) - Facet joint
neurolysis, subsequent levels

xxiii. Thoracic paravertebral facet joint nerve neuroly sis (T5/6 joint - T5 and T6 medial branch nerves) 2000 Codes:
$64626-\mathrm{C} / \mathrm{T}$ paravertebral facet joint neurolysis, single level (T5/6 joint)
76005 - Fluoroscopic guidance
1999 Codes:

No specific codes are available (lumbar neurolysis codes are used)
64622 - Facet joint neurolysis, single level

xxiv. Multiple thoracic paravertebral facet joint neu rolysis (T4/5 through T6/7 joints - T4 through T7 medial branches)
$64626-\mathrm{C} / \mathrm{T}$ paravertebral facet joint neurolysis, single level (T4/5 joint)
64627-51 (two units) - C/T paraverte bral facet joint neurolysis, each additional level (T5/6 and T6/7 joints) 1999 Codes:

No specific codes are available (lumbar neurolysis codes are used)
- 64622 - Facet joint neurolysis, single level
64623-51 (two units) - Facet joint neurolysis, subsequent levels

Xxv. Lumbar paravertebral facet joint neurolysis (L4/ 5 joint - L3 and L4 medial branch nerves)

\section{Codes:}

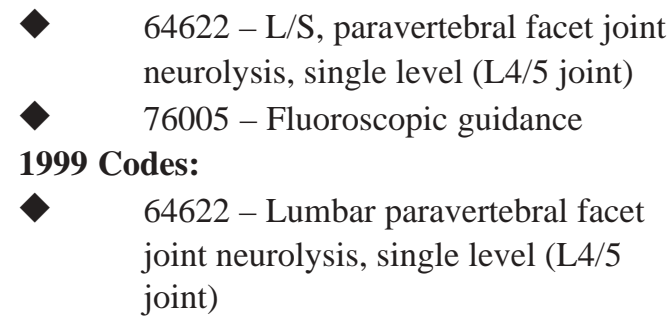

xxvi. Multiple lumbar paravertebral facet joint neuroly sis (L3/4 through L5/S1 joints L2 through L4 medial branch nerves and L5 dorsal ramus) 2000 Codes:

- 64622 - L/S paravertebral facet joint neurolysis, single level (L3/4 joint)

64623-51 (two units) - L/S paravertebral facet joint neurolysis, eachadditional level (L4/5 and L5/S1 joints)

76005 - Fluoroscopic guidance 1999 Codes:
- 64622 - Lumbar paravertebral facet joint neurolysis, single level (L3/4 joint) 64623-51 (two units) - Lumbar paravertebral facet joint neurolysis, each additional level (L4/5 and L5/S1 joints)

xxvii. Bilateral lumbar facet joint neurolysis (L3/4 through L5/S1 joints - L2 through L4 medial branch nerves and L5 dorsal ramus)

2000 Codes:

64622-50 - L/S paravertebral facet joint neurolysis, single level (L3/4 joint bilateral)

64623-50 - L/S paravertebral facet joint neurolysis, each additional level (L4/5 and L5/S1 joint bilateral)

76005 - Fluoroscopic guidance 1999 Codes:

Bilateral billing is not available for 1999 CPT codes

64622 - Facet joint neurolysis, single
level (L3/4 joint)
64623-51 (two units) - Facet joint
neurolysis, each additional level (L4/5
and L5/S1 joints)




\section{EPIDURAL INJECTIONS}

\section{Procedure (CPT) Codes}

1999 Codes (6)

$\checkmark 62275$ - Injection of diagnostic or therapeutic anesthetic or antispasmodic substance (including narcotics); epidural, cervical or thoracic, single

$\checkmark 62278$ - Injection of diagnostic or therapeutic anesthetic or antispasmodic substance (including narcotics); epidural, lumbar or caudal, single

$\checkmark 62279$ - Injection of diagnostic or therapeutic anesthetic or antispasmodic substance (including narcotics); epidural, lumbar or caudal, continuous

$\checkmark 62289$-Injection of substance other than anesthetic, antispasmodic, contrast, or neurolytic solutions; lumbar or caudal (separate procedure)

- 62298 - Injection of substance other than anesthetic, contrast, or neurolytic solutions, epidural, cervical or thoracic (separate procedure)

$\checkmark 62284-$ Injection procedure for myelography

2000 Codes (5)

- 62310 - Injection, single (not via indwelling catheter), not including neurolytic substances, with or without contrast (for either localization or epidurography), of diagnostic or therapeutic substance(s) (including anesthetic, antispasmodic, opioid, steroid, other solution), epidural or subarachnoid; cervical or thoracic

- 62311 - Injection, single (not via indwelling catheter), not including neurolytic substances, with or without contrast (for either localization or epidurography), of diagnostic or therapeutic substance(s) (including anesthetic, antispasmodic, opioid, steroid, other solution), epidural or subarachnoid; lumbar, sacral (caudal)

- 62318 - Injection, including catheter placement, continuous infusion or intermittent bolus, not including neurolytic substances, with or without contrast (for either localization or epidurography), of diagnostic or therapeutic substance(s) (including anesthetic, antispasmodic, opioid, steroid, other solution), epidural or subarachnoid; cervical or thoracic

62319 - Injection, including catheter place-

ment, continuous infusion or intermittent bolus, not including neurolytic substances, with or without contrast (for either localization or epidurography), of diagnostic or therapeutic substance(s) (including anesthetic, antispasmodic, opioid, steroid, other solution), epidural or subarachnoid; lumbar, sacral (caudal)

- 64479 - Injection, anesthetic agent and/or steroid, transforaminal epidural; cervical or thoracic, single level

- 64480 - Injection, anesthetic agent and/or steroid, transforaminal epidural; cervical or thoracic, each additional level

- 64483 - Injection, anesthetic agent and/or steroid, transforaminal epidural; lumbar or sacral, single level

- 64484 - Injection, anesthetic agent and/or steroid, transforaminal epidural; lumbar or sacral, each additional level

$\checkmark 72275$ - Epidurography, radiological supervision and interpretation

- 76005 - Fluoroscopic guidance and localization or needle or catheter tip for spine or paraspinous diagnostic or therapeutic procedures

\section{Examples}

i. Cervical interlaminar epidural injection - with out fluoroscopy

2000 Codes:

$\checkmark \quad 62310-\mathrm{C} / \mathrm{T}$ epidural

1999 Codes:

- 62275 or $62298-\mathrm{C} / \mathrm{T}$ epidural

ii. Cervical interlaminar epidural injection - with fluoroscopy

2000 Codes:

- $62310-\mathrm{C} / \mathrm{T}$ epidural

- 76005 - Fluoroscopic guidance 1999 Codes:

$\checkmark \quad 62275$ or $62298-\mathrm{C} / \mathrm{T}$ epidural

iii. Thoracic interlaminar epidural injection - with out fluoroscopy

2000 Codes:

$\checkmark 62310-\mathrm{C} / \mathrm{T}$ epidural

1999 Codes:

- 62275 or $62298-\mathrm{C} / \mathrm{T}$ epidural

iv. Thoracic interlaminar epidural injection - with fluoroscopy 
2000 Codes:

$$
\begin{aligned}
& 62310-\text { C/T epidural } \\
& 1999 \text { Codes: } \\
& \quad 62275 \text { or } 62298-\text { C/T epidural }
\end{aligned}
$$

v. Lumbar interlaminar epidural injection - without fluoroscopy

\section{Codes:}

$62311-$ L/S epidural

\section{Codes:}

62278 or62289 - Lumbar epidural

vi. Lumbar interlaminar epidural - with fluoroscopy 2000 Codes:

$$
\begin{aligned}
& 62311 \text { - L/S epidural } \\
& 76005 \text { - Fluoroscopic guidance } \\
& 1999 \text { Codes: } \\
& 62278 \text { or } 62289 \text {-Lumbar epidural }
\end{aligned}
$$

vii. Caudal epidural injection - without fluoroscopy

\section{Codes:}

$$
\begin{aligned}
& \text { 62311 - L/S epidural } \\
& 1999 \text { Codes: } \\
& \text { epidural } 62278 \text { or } 62289 \text { - Lumbar/caudal }
\end{aligned}
$$

viii. Caudal epidural injection - with fluoroscopy

\section{Codes:}

$$
\begin{aligned}
& 62311 \text { - L/S epidural } \\
& 76005 \text { - Fluoroscopic guidance } \\
& 1999 \text { Codes: } \\
& 62278 \text { or } 62289 \text {-Lumbar/caudal } \\
& \text { epidural }
\end{aligned}
$$

ix. Cervical transforaminal epidural injection, single level (C5 spinal nerve, C4/5 foramen)

\section{Codes:}

$$
\begin{aligned}
& 64479-\mathrm{C} / \mathrm{T} \text { transforaminal epidural, } \\
& \text { single level (C5) } \\
& 76005 \text { - Fluoroscopic guidance }
\end{aligned}
$$

1999 Codes: (Separate transforaminal codes are not available)

62275 or $62298-\mathrm{C} / \mathrm{T}$ epidural

x. Cervical transforaminal epidural injection, mul tiple levels (C6 and C7 spinal nerves)

\section{Codes:}

- $64479-\mathrm{C} / \mathrm{T}$ transforaminal epidural, single level (C6)

64480-51 - C/T transforaminal epidural, each additional level (C7) 76005 - Fluoroscopic guidance

1999 Codes: (Separate transforaminal codes are not available)

62275 or $62298-\mathrm{C} / \mathrm{T}$ epidural

xi. Thoracic transforaminal or selective epidural injection, single level (T5 spinal nerve, T4/5 foramen)

\section{Codes:}

- $64479-\mathrm{C} / \mathrm{T}$, transforaminal epidural, single level (T5)

76005 - Fluoroscopic guidance

1999 Codes: (Separate transforaminal codes are not available)

62275 or $62298-\mathrm{C} / \mathrm{T}$ epidural

xii. Thoracic transforaminal or selective epidural in jection, multiple levels (T6 and T7 spinal nerves) 2000 Codes:

$64479-\mathrm{C} / \mathrm{T}$, single level (T6)

64480-51 - C/T transforaminal epidural, each additional level (T7)

76005 - Fluoroscopic guidance 1999 Codes: (Separate transforaminal codes are not available)

62275 or $62298-\mathrm{C} / \mathrm{T}$ epidural

xiii. Bilateral thoracic transforaminal or selective epidural injections (T5, T6, and T7 spinal nerves) 2000 Codes:

64479-50 - CT transforaminal
epidural, single level (T5 bilateral)
$64480-50-\mathrm{C} / \mathrm{T}$ transforaminal
epidural, each additional level (T6, T7
bilateral)
76005 - Fluoroscopic guidance
1999 Codes: (Separate transforaminal codes are
not available)
62275 or $62298-\mathrm{C} / \mathrm{T}$ epidural

xiv. Lumbar transforaminal or selective epidural injection (L5 spinal nerve) 


\section{Codes:}

64483 - L/S transforaminal epidural, single level (L5)

76005 - Fluoroscopic guidance 1999 Codes: (Separate transforaminal codes are not available)

62278 or 62289 - Lumbar epidural

Xv. Lumbar transforaminal or selective epidural injection, multiple levels (L4, L5, and S1 spinal nerves)

2000 Codes:

64483 - L/S transforaminal epidural, single level (L4)

64484-51 (two units) - L/S

transforaminal epidural, each

additional level (L5 and S1)

76005 - Fluoroscopic guidance

1999 Codes: (Separate transforaminal codes are not available)

62278 or 62289 - Lumbar epidural

xvi. Bilateral lumbar transforaminal or selective epidural injections (L5, S1)

2000 Codes

64483-50 - L/S transforaminal epidural, single level (L5 bilateral)

64484-50 - L/S transforaminal epidural, each additional level (S1 bilateral)

76005 - Fluoroscopic guidance

1999 Codes: (Separate transforaminal codes are not available)

- 62278 or 62289 -Lumbar epidural

\section{PERCUTANEOUS LYSIS OF EPIDURAL ADHESIONS}

\section{Procedure (CPT) Codes} 1999 Codes (6)

62281 - Injection of neurolytic substance (eg, alcohol, phenol, saline solutions); epidural, cervical or thoracic

62282 - Injection of neurolytic substance (eg, alcohol, phenol, saline solutions); epidural, lumbar or caudal
62284 - Injection procedure for myelography and/or computerized axial tomography, spinal (other than C1-2 and posterior fossa)

64714 - Neuroplasty, major peripheral nerve, lumbar plexus or 64722 , decompression; unspecified nerve(s) or

\section{Code (5)}

62263 - Percutaneous lysis of epidural adhesions using solution injection (eg, hypertonic saline, enzyme) or me chanical means (eg, springwirecatheter) including radiologic localization (includes contrast when administered)

\section{Examples}

Percutaneous lysis of adhesions utilizing Racz catheter 2000 Codes:

62263 - Percutaneous lysis of epidural adhesions

1999 Codes: (Specific codes(s) are not available)

62282 - Lumbar neurolytic block

64714 - Neuroplasty, lumbar plexus

22899 - Unlisted, spine procedure

Percutaneous lysis of adhesions - with spinal endoscopy 2000 Codes:

62263 - Percutaneous lysis of epidural adhesions

22899 - Unlisted, spine procedure

64714 - Neuroplasty, lumbar plexus 1999 Codes:

64714 - Neuroplasty, lumbar plexus 22899 - Unlisted, spine procedure

\section{DISCOGRAPHY AND ANNULOPLASTY}

\section{Procedure (CPT) Codes (5, 6)}

62290 - Injection procedure for discography, each level; lumbar 62291 - Injection procedure for discography, each level; cervical or thoracic 
No CPT codes are available for disc thermal annuloplasty. The following code is used with revised description:

62287 - Aspiration or decompression procedure, percutaneous, of nucleus pulposus of intervertebral dis $\mathrm{k}$, a n y method, single or multiple levels, lumbar (eg, manual or automated percutaneous diskectomy, percutaneous laser diskectomy)

Example(s)

i. Cervical discography - multiple levels (C4/5, C5/6)

$62291-\mathrm{C} / \mathrm{T}$ discography, each level (C4/5)

62291-51 - C/T discography, each level (C5/6)

72285-26 - C/T discography, radio logical supervision and interpretation

ii. Thoracic discography - multiple levels (T4/5, T5/6)

$$
\begin{aligned}
& \text { 62291-C/T, each level (T4/5) } \\
& \text { 62291-51 - C/T, each level (T5/6) } \\
& \text { 72285-26 - C/T discography, radio } \\
& \text { logical supervision and interpretation }
\end{aligned}
$$

iii. Lumbar discography - multiple levels (L3/4, L4/5, L5/S1)

$$
\begin{aligned}
& 62290 \text { - Lumbar discography, each } \\
& \text { level (L3/4) } \\
& 62290-51 \text { - Lumbar discography, each } \\
& \text { level (L4/5, L5/S1) } \\
& \text { 72295-26 - Lumbar discography, } \\
& \text { radiological supervision and } \\
& \text { interpretation }
\end{aligned}
$$

iv. Intradiscal electric thermal annuloplasty, single or multiple levels

$$
\begin{aligned}
& 62287 \text { - Aspiration or decompression } \\
& \text { procedure, percutaneous, of nucleus } \\
& \text { pulposus of intervertebral disk, any } \\
& \text { method, single or multiple levels } \\
& 22899 \text { - Unlisted, spine procedure }
\end{aligned}
$$

\section{EPIDUROGRAPHY}

\section{Code:}

72275 - May be used during first epidural procedure

1999 Code: (No specific code available, myelography code is used instead)

$$
\begin{aligned}
& 62284-\text { May be used during first } \\
& \text { epidural procedure }
\end{aligned}
$$

\section{MULTIPLE REGIONS AND/OR COMBINATIONS}

i. Multiple cervical facet joint nerve blocks, (C3/4-C5/6 joints - C3-6 medial branch nerves) and lumbar facet joint nerve blocks, (L3/4-L5/ $\mathrm{S} 1$ joints L2-L4 medial branches and L5 dorsalramus)

\section{Codes:}

- $64470-\mathrm{C} / \mathrm{T}$ facet joint nerve block, single level (C3/4 joint)

64472-51 - C/T facet joint nerve blocks, each additional level (C4/5 joint)

64475-51 - L/S facet joint nerve block, single level (L3/4 joint)

64476-51 (two units) - L/S facet joint nerve blocks, each additional level (L4/5 joint)

76005 Fluoroscopic guidance (may or may not be used)

1999 Codes: (No cervical codes are available. Lumbar codes are used for cervical)

$$
\begin{aligned}
& 64442 \text { - Facet joint nerve block, } \\
& \text { single level } \\
& 64443-51 \text { (two or possibly three units) } \\
& \text { - Facet joint nerve blocks, subsequent } \\
& \text { levels }
\end{aligned}
$$

ii. Caudal epidural and multiple cervical facet joint nerve blocks (C3/4-C5/6 joints - C3-6 medial branch nerves)

\section{Codes:}

$62311-$ L/S epidural
$64470-51-$ CT facet joint nerve block,
single level
64472-51 (two units) - C/T facet joint
nerve blocks, additional levels
76005 - Fluoroscopic guidance


1999 Codes: (No cervical facet joint nerve block codes are available. Lumbar codes are used)

62289 or 62278 - Caudal epidural

64442-51 - Facet joint nerve block, single level

64443-51 (two units) - Facet joint nerve blocks, subsequent levels

iii. Cervical epidural and multiple lumbar facet joint nerve blocks

2000 Codes:

$62310-$ Cervical epidural

64475-51 - L/S facet joint nerve block, single level

64476-51 - L/S facet joint nerve blocks, additional levels

1999 Codes:

62275 or 62298 - Cervical epidural

64442-51 - Lumbar facet joint nerve block, single level

64443-51 - Lumbar facet joint nerve blocks, subsequent levels

iv. Multiple cervical transforaminal epidurals and lumbar facet joint nerve blocks

2000 Codes:

64479-Cervical transforaminal, single level

64481-51 - Cervical transforaminal, each additional level

64475-51 - L/S facet joint nerve block, single level

64476-51 - L/S facet joint nerve blocks, each additional level

76005 - Fluoroscopic guidance

1999 Codes: (No transforaminal epidural codes are available. Cervical epidural codes are used) 62275 or 62298 - Cervical epidural 64442-51 - Lumbar facet joint nerve block, single level 64443-51 - Lumbar facet joint nerve blocks, subsequent levels

v. Multiple lumbar transforaminal epidurals and multiple cervical facet joint nerve blocks

2000 Codes:

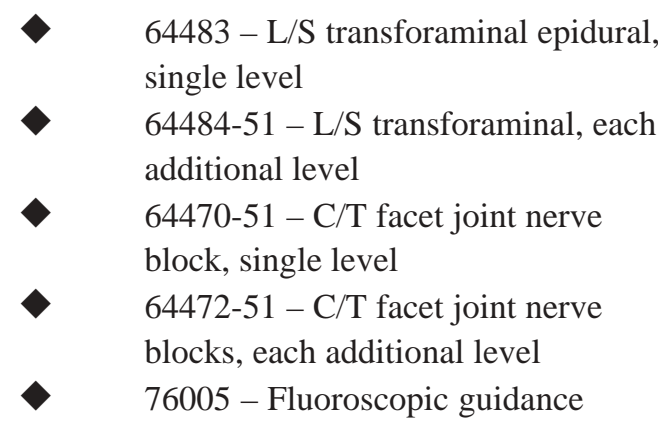

1999 Codes: (No transforaminal or cervical facet joint nerve block codes are available. Lumbar epidural and facet joint nerve block codes are used)

62289 or 62278 - Lumbar epidural

64442-51 - Lumbar facet joint nerve block, single level

64443-51 (two units) - Lumbar facet joint nerve blocks, subsequent levels

vi. Percutaneous lysis of adhesions and multiple cervical facet joint nerve blocks

\section{Codes:}

62263 - Percutaneous lysis of epidural adhesions

64470-51 C/T facet joint nerve block, single level

64472-51 (two units) - C/T facet joint nerve blocks, each additional level 76005 Fluoroscopic guidance

1999 Codes: (No percutaneous lysis or cervical facet joint nerve block codes are available. Lumbar neurolytic and lumbar facet joint nerve block codes are used)

62282 - Lumbar neurolytic epidural

64442-51 - Facet joint nerve block, single level

64443-51 (two units) - Facet joint nerve blocks, subsequent levels

\section{CONCLUSION}

It is accepted that CPT descriptive terms and identification of codes currently are the only means of documenting medical necessity and serve a wide variety of important functions in the field of medical practice including interventional pain management. Documentation of interventional pain procedures and subsequent billing and coding are of crucial importance not only in complying 
with the regulations, but also for good patient care. This review has provided generally accepted practice patterns in a safe manner.

\section{REFERENCES}

1. Butler L. Clinton budget boosts efforts to combat Medicare fraud. American Med News 2000; 43: 1, 34, 35.

2. Klein SA. Feds try new talk in charging doctors with upcoding. American Med News 2000; 43: 5-6.

3. International Classification of Diseases, Ninth Revision, Clinical Modification, ICD-9-CM. Reston, St. Anthony Publishing, 1998.

4. Manchikanti L. Role of evaluation and management services in pain management. Pain Physician 1999; 2:10-32.

5. Current Procedural Terminology, CPT 2000, Chicago, American Medical Association, 1999.
6. Current Procedure Terminology, CPT 1999. Chicago, American Medical Association, 1998.

7. Manchikanti L. CPT 2000. Interventional pain management coding in the new millennium. Pain Physician 2000; 3:73-85.

8. Manchikanti L, Singh V, Bakhit CE et al. Interventional techniques in the management of chronic pain. Part 1.0. Pain Physician 2000; 3:7-42.

9. 2000 Common Coding Scenarios for Spine Procedures and Injection Techniques. Rosemont, IL, North American Spine Society, 2000, pp 1-55.

10. Manchikanti L. Impact of national correct coding policy on interventional pain management. Pain Physician 1999; 2:33-45.

11. The National Correct Coding Manual - version 5.0. Alpharetta, GA, The Medical Management Institute, pp. $1-239$.

12. Physician Practice Coder 2000. 6: March, pp 3-4. 